\title{
LA SITUACIÓN DEL P.A.S FUNCIONARIO
}

\section{THE STATUS OF THE OFFICER P.A.S}

\section{AUTORES}

Rafael Catalá Mateo: Ex-miembro de la Junta de Personal (PAS funcionario) durante 8 años

rafael.catala@ozu.es

\section{CURRÍCULUM VITAE}

Jefe de Sección de Becas en el servicio de alumnos y planes de estudio en la Universidad de Alcalá de Henares (España).

\section{RESUMEN}

La Relación de Puestos de Trabajo (en adelante RPT) del Personal de Administración y Servicios funcionario (en adelante PAS funcionario) identifica y clasifica todos los puestos de trabajo del mencionado colectivo según una serie de aspectos como unidades administrativas en que se integran, denominación, $\mathrm{n}^{\mathrm{o}}$ de plazas, condiciones generales del puesto, nivel de dedicación, etc.; es por tanto, la columna vertebral sobre la que recae todo el peso de la gestión administrativa de nuestra Universidad, lo cual le confiere una cierta entidad dentro de nuestra Institución.

\section{PALABRAS CLAVE}

PAS - RPT - Puestos de trabajo - Personal de administración - Servicios funcionario

\section{ABSTRACT}


The Jobs Ratio (hereafter RPT) of the Administration and Services Officer (hereinafter PAS official) identifies and classifies all jobs in the group mentioned by a number of issues as administrative units to which they belong, name, number of seats, general conditions of the job, level of dedication, and so on. it is therefore the backbone upon which rests the whole weight of the administrative management of our University, which gives it a certain entity within our institution.

\section{KEY WORDS}

PAS - RPT - Jobs - Administrative Staff - Services officer

\section{LA SITUACIÓN DEL P.A.S FUNCIONARIO} ¿UN CALLEJÓN SIN SALIDA?

En el momento de sacar esta edición, hemos recibido en la redacción, del autor de este mismo artículo, una nota de última hora que incluimos a continuación:

NOTA DE ÚLTIMA HORA:

SEGÚN PARECE, EN LA REUNIÓN CELEBRADA ENTRE LA GERENCIA Y LA JUNTA DE PERSONAL EN LA TARDE DEL PASADO DÍA 12 DE JULIO DE 1999, SE HA PRODUCIDO ALGÚN AVANCE QUE PUEDE DESBLOQUEAR, DESPUÉS DE VARIOS MESES, ALGUNOS DE LOS ASPECTOS MÁS CONFLICTIVOS DE LA NEGOCIACIÓN. ESPERAMOS QUE PUEDA SER EL PUNTO DE PARTIDA PARA CONSEGUIR UN CONSENSO QUE SEA ACEPTADO POR TODA LA PLANTILLA. 
Rafael Catalá Mateo.

La Relación de Puestos de Trabajo (en adelante RPT) del Personal de Administración y Servicios funcionario (en adelante PAS funcionario) identifica y clasifica todos los puestos de trabajo del mencionado colectivo según una serie de aspectos como unidades administrativas en que se integran, denominación, $n^{\circ}$ de plazas, condiciones generales del puesto, nivel de dedicación, etc.; es por tanto, la columna vertebral sobre la que recae todo el peso de la gestión administrativa de nuestra Universidad, lo cual le confiere una cierta entidad dentro de nuestra Institución.

Hasta hace aproximadamente 6 años, fecha en la que se produjo un cambio en la Gerencia, todas las propuestas de modificación de la RPT, se aprobaban por los diferentes órganos de la Universidad con el acuerdo entre los órganos de representación del PAS funcionario y la Gerencia, todo ello con el consiguiente esfuerzo y acercamiento de posturas por ambas partes en, a veces, largos procesos de negociación. Sin embargo, desde aquellas fechas no se ha producido ningún acuerdo en una modificación sustancial de la RPT del personal funcionario, que a estas alturas, resulta ya imprescindible por el deterioro que ha sufrido esta estructura administrativa a lo largo de estos últimos años (estancamientos salariales, fomento de comisiones de servicio en puestos no creados, proliferación de la contratación temporal, funciones y tareas poco claras, estructuras administrativas obsoletas, etc. ).

En estos momentos, el PAS funcionario se encuentra inmerso, una vez más, en un proceso de negociación de una nueva RPT, y para no romper con lo que viene siendo tradicional en los últimos años, el proceso no avanza, por no decir que se encuentra prácticamente bloqueado. 
Pueden existir muchos factores que expliquen esta situación, sin embargo, desde mi punto de vista, me atrevería a señalar algunos, consciente de que me dejo aspectos importantes en el tintero:

1.- A pesar de que estatutariamente, la elaboración de la plantilla orgánica corresponde a la Gerencia, aspecto que nadie cuestiona, supone un error el hacerlo completamente al margen de los trabajadores, sin tener en cuenta sus necesidades, inquietudes, problemas o propuestas que puedan plantarse desde aquellos que forman las unidades y servicios de la Universidad, que en la mayor parte de las ocasiones la primera noticia que tienen de la propuesta de la Gerencia es la que les facilita la Junta de Personal como órgano de representación correspondiente. Esto provoca un alejamiento muy negativo entre quienes dirigen y planifican la gestión administrativa y aquellos que la tienen que poner en práctica, sobre todo cuando suponen en algunas cuestiones una "vuelta de tuerca" más en el deterioro de las condiciones de trabajo.

2.- A su vez, resulta muy negativa la actitud de la Gerencia y del Rector ante el proceso de negociación que, en ningún caso es entendido como un derecho legítimo de los trabajadores sino como una traba para la consecución de sus fines, de ahí el reiterado argumento del Rector de que los órganos de gobierno de la Universidad (Junta de Gobierno, Consejo Social) no tienen que negociar nada, sino que aprueban o no en función de las votaciones correspondientes; esto último, si bien es cierto, no excluye que la administración que él dirige debería negociar e intentar conseguir acuerdos para no trasladar los conflictos a los citados órganos. Son planteamientos como los señalados los que fomentan actitudes inmovilistas ante las propuestas y contrapropuestas que sistemáticamente se han venido realizando desde los órganos de representación de los funcionarios, y que en ningún caso, han logrado modificar las posturas iniciales de la Gerencia que prefiere, como ha ocurrido en muchas 
ocasiones, retirar su propuesta y mantener indefinida una situación que en muchos casos resulta ya insostenible.

3.- Por último, mencionaría una falta de capacidad para la negociación que, en principio, podría achacarse tanto a la Gerencia como a la Junta de Personal, sin embargo, este órgano de representación ha cambiado en los últimos años varias veces, su composición, sus miembros, sus cargos e, incluso, las fuerzas sindicales que conforman sus mayorías y, no obstante, los resultados de las negociaciones siguen siendo nulos, puesto que una de las partes no modifica en nada sus propuestas, ni cede lo más mínimo.

Esta situación, prolongada en los últimos seis años, acarrea una serie de consecuencias muy negativas de las cuales destacaría dos:

En primer lugar, se ha ido perdiendo, en la plantilla el "principio de pertenencia", cuya principal consecuencia es no identificar los objetivos de la Universidad como propios; cada vez para un mayor número de trabajadores la Universidad se ha convertido en una fábrica donde se trabaja unas horas a cambio de un salario, sin que exista una relación que vaya más allá de la estrictamente laboral, exceptuando, como es lógico, los cada vez más abundantes puestos de libre designación o de confianza y aquellos otros que obtienen, a corto plazo, un interés personal que anteponen al del resto del colectivo.

Somos cada vez más los trabajadores que añoramos el ambiente de trabajo de hace algunos años, en donde se cuidaban y fomentaban las relaciones humanas más allá de los actuales principios de eficacia, eficiencia, reducción de costes, etc. y que se caracterizaban por un entusiasmo y una disposición inexistentes en la actualidad. Todo ello ha creado un ambiente de crispación y tensión desconocido en la Universidad de Alcalá. 
En segundo lugar, lo expuesto hasta ahora ha provocado un deterioro en la gestión administrativa que, aunque de momento no ha afectado a los objetivos básicos de la Institución, no sería de extrañar que en el futuro se presenten situaciones de difícil solución, puesto que el cumplimiento de algunos objetivos esenciales no puede justificar reiteradamente los métodos empleados, en muchos casos porque dichos métodos son totalmente contrarios a los principios de eficacia y eficiencia que los propios gestores abanderan.

En estos momentos, sin embargo, la Gerencia y el Rector parecen estar dispuestos a seguir adelante con la modificación de la RPT, a pesar de no contar con el apoyo de la inmensa mayoría de la plantilla, trasladando el problema a la Junta de Gobierno y al Consejo Social, en donde, si la fuerza de los votos lo consigue, podrán imponerla al PAS funcionario.

No obstante, y para finalizar, si esta hipótesis llegase a producirse, alguien debería meditar sobre sus consecuencias. Una RPT impuesta por la fuerza de los votos y no asumida por el PAS funcionario, podrá ser legítima, pero estará, muy probablemente, abocada al fracaso pues jamás podrá contar con la voluntad, motivación e implicación mínima de los trabajadores para que funcione, y además, abriría, quizás de forma irreversible, la brecha existente entre la Gerencia y la plantilla. 